\title{
LES CONSEQUENCES DE TCHERNOBYL EI LA SOCIOPATHOLOGIE
}

\section{Laurent Bocéno}

\section{Université de Caen Basse-Normandie - France}

Résumé: Dans la situation de post-catastrophe aux alentours de Tchernobyl, la vie quotidienne est une correspondance constante entre l'institué et le vécu qui sont souvent analysés séparément. Avec l'approche par les sociopathologies, il est possible d'examiner le social entré dans le corps comme le résultat de l'intériorisation en nous, d'archétypes sociaux d'une part et d'autre part, de situations incorporées. Les sociopathologies sont liées aux formes d'institutionnalisation dont le sens est souvent ignoré par les personnes qui y sont confrontées.

Mots clés: contamination, radioactivité, sociopathologie, Tchernobyl.

Resumo: Na situação de pós-catástrofe nos arredores de Chernobyl, a vida cotidiana é uma correspondente constante entre o instituído e o vivido, que com freqüência são analisados de forma separada. Sob o prisma das sociopatologias, é possível examinar o social entrando no corpo como resultado da interiorização, em nós, dos arquétipos sociais por um lado, e, por outro lado, de situações incorporadas. As sociopatologias são ligadas às formas de institucionalização cujo sentido é seguidamente ignorado pelas pessoas confrontadas nesse processo.

Palavras-chave: Chernobyl, contaminação, radioatividade, sociopatologia.

Cet article présente une part des conclusions d'enquêtes menées, entre 1997 et 2001, sur les territoires contaminées par l'explosion du réacteur “4” de la centrale nucléaire Lénine de Tchernobyl, en 1986. Notre terrain est situé en République du Bélarus où l'environnement présente une forte contamination radioactive artificielle consécutive de l'explosion de Tchernobyl qui est implanté dans un pays frontalier, l'Ukraine. C'est dans le cadre d'une étude financée par la Communauté Européenne que nous avons été amené à travailler au Bélarus, ancienne république soviétique, pays ayant subi le plus fortement les retombées du nuage radioactif. Notre travail initial consistait, pour des chercheurs suisses et hollandais, à collecter des données factuelles afin d'élaborer un 
programme d'éducation à la santé destiné aux enfants des territoires contaminés par l'explosion de Tchernobyl (Gerirad, 1998).

L’objectif de ce programme était de limiter les effets délétères de la radioactivité sur la santé. A partir de 1997, nous avons rencontré, au Bélarus, des autorités nationales, régionales et locales afin de pouvoir, non sans difficultés parfois, mener des entretiens auprès des populations: chez l'habitant, dans des écoles, dans des hôpitaux, dans la rue, etc. Si notre travail initial a d'abord été mené dans les régions rurales du sud-est du pays vers les frontières avec la Russie et l'Ukraine, nous avons pourtant parcouru l'ensemble du pays pour suivre les itinéraires d'habitants déplacés et pour rencontrer des équipes de scientifiques, de soignants travaillant sur les conséquences sanitaires de Tchernobyl. Nous ne nous sommes pas limité aux cadres définis par les missions officielles dans un pays où le régime politique limite la liberté d'expression et d'opinion. De manière indépendante, nous avons rencontré, lors d'autres voyages, des médecins, des scientifiques, des journalistes ou des habitants critiques tant par rapport aux thèses défendues par les représentants de l'administration du Bélarus que par les institutions internationales comme l'Agence Internationale pour l'Energie Atomique (AIEA) et l'Organisation Mondiale pour la Santé (OMS). Cette indépendance nous a permis de nous défaire, formellement et symboliquement, de la tutelle de l'administration du Bélarus et d'accéder à d'autres discours ou démonstrations que ceux préconisés par des autorités cherchant en permanence à inscrire dans un univers institutionnel définitivement passé, celui de l’URSS, les nouvelles formes émergentes du "vivre ensemble” initialisées par Tchernobyl. En effet, les recours classiques et traditionnels ne sont plus suffisant pour réduire la vulnérabilité et l'indétermination consécutives de la catastrophe nucléaire et voulons illustrer, à l'aide de la notion de sociopathologie, une part de la complexité sociale à l'œuvre dans les territoires contaminés par la radioactivité artificielle (Bocéno, 2005).

Notre approche se fonde sur la tradition sociologique française indissociable des figures de Émile Durkheim $(1960,1981)$ et de Marcel Mauss (1968). Nous ne reprenons pas, ici, l'ensemble des arguments conceptuels qu'il faudrait convoquer mais nous tenons à préciser qu'il s'agit, au terme d'une démarche heuristique, par une observation flottante opaque, de souligner en quoi les sociopathologies sont des éléments constitutifs des sociétés. Il s’agit ici d’une contribution partielle à l'élaboration du concept de sociopathologie qui permet, selon nous, de mieux comprendre les dynamiques sociales qui, soit par le recours au champ conjoint de la médecine et de la science expérimentale, soit par les 
références à la tradition, soit par l'usage des appareils industriels et technocrates en charge de la gestion politique et économique des catastrophes mettent en place des dynamiques d'incorporation et d'intégration institutionnelles.

Soulignons que la délimitation entre les référentiels possibles est réservée à l'usage de la démonstration et que l'imbrication organisant les sociopathologies en "faits sociaux totaux" tel que les a conceptualisé Marcel Mauss, puise nécessairement dans tous les registres du social. Il faut donc, par exemple, mobiliser toute la dimension anthropologique pour accéder à la compréhension des jeux de mémoire: qu'il s'agisse de nostalgie relative à la disparition d’un mode de vie traditionnel sous de nouvelles règles et normes coercitives (enterrement de villages et de maisons, etc.; de plaintes face aux développements de maladies, de difficultés sociales, etc.; des rituels de deuil, etc. ${ }^{1}$ En convoquant le concept de sociopathologie, l'intérêt est de travailler sur le social dans les corps, sur la façon dont "le social est incorporé et institué dans les corps, dans les prothèses ou les appareils institutionnels porteurs de signes d'une humanité qui cherche à atténuer, réduire ou dissimuler leurs propres influences pathogènes”.

A partir d'évènement comme Tchernobyl et la mise en perspective des effets de pollution environnementale, il est possible de considérer le traitement social de la pathologie comme étant d'autant plus spécifique qu'elle présente un caractère d'incorporation élevé. ${ }^{2}$ Et là, ce qui relève de l'ordinaire et du quotidien peut avoir des conséquences sanitaires parfois graves (Juan, 2001). Avec Tchernobyl, nous sommes face à des pathologies pouvant, à un moment donné, être qualifiées d'évènementielles. Pour les saisir, les outils théoriques élaborés par Kleinman $(1980,1988)$ sont utiles car ils permettent de considérer la pathologie et ses systèmes explicatifs dans une relation avec des cadres culturels dans une perspective de compréhension: soit comme une expérience culturelle subjective (Illness), soit comme une catégorie médicale ou traditionnelle (Disease) ou soit encore comme un objet social (sickness) lorsqu'elle atteste et acquière un statut social légitime. Ainsi Kleinman permet d'analyser la rationalisation des conduites des individus dans un cadre culturel

1 Soulignons d'ailleurs qu'avec la ritualisation, il ne s'agit rien de moins que d'un rappel que si l'individu est bien dans le monde social, ce dernier est aussi dans le corps qui parfois peut être honoré malgré son absence sous la dalle funéraire.

2 Mais il ne faut pas en déduire que le concept sociopathologie fasse toujours appel de la catastrophe tant il existe des sociopathologies, sans catastrophe, sans évènement fondateur, qui dépourvues de labellisation officielle et clinquante n'en sont pas pour autant inoffensive.

Horizontes Antropológicos, Porto Alegre, ano 12, n. 25, p. 191-216, jan./jun. 2006 
spécifique et en observer les interactions avec le système sanitaire ou ses représentants. Mais il ne traite pas spécifiquement la dimension instituant la pathologie, celle qui est liée à la dynamique d'incorporation pathogène comme à la perception de celle-ci par l'individu qui, en dernier ressort et comme acteur, apporte une touche déterminante à la dimension sociale des pathologies.

Cette dimension est intéressante car elle indique que, dans le cas de Tchernobyl, si le cadre culturel change, l'activité de construction et d'institutionnalisation des pathologies reste bien un outil de gestion des conséquences sanitaires, économiques et sociales. Et à cette élaboration participent, à des degrés divers, tant les responsables politiques que les technocrates nucléaristes, leurs opposants que les victimes, informées ou non de leur statut. C'est pourquoi, il nous semble intéressant de présenter brièvement un panorama problématique lié au développement des techno-sciences qui, s’il est relativement récent dans la perception du risque environnemental, bouscule pourtant radicalement les perspectives humaines.

\section{Un nouveau monde s'ouvre avec Thernobyl}

C'est avec une perception nouvelle des catastrophes technologiques que, dans les années 1980, le monde et particulièrement l'occident, se découvrent producteurs et promoteurs, conscients ou non, de nouvelles formes de réalités. Dans la conscience collective occidentale s'impriment les évènements des graves pollutions environnementales comme dans les cas les plus connus de Seveso (Italie) avec des rejets de dioxine en 1976, avant que l'année 1984 soit marquée par la contamination de l'usine chimique de Bhopal (Inde) et la menace d'explosion nucléaire dans la centrale de Three Miles Island (USA) et encore concernant l'industrie nucléaire l'explosion de la centrale soviétique de Tchernobyl, en 1986.

Tchernobyl semble être la marque de l'altération du crédit accordé au développement des techno-sciences. En effet, l'explosion de la centrale soviétique de Tchernobyl devient un repère donnant toute son importance à l’ouvrage de Hans Jonas (1990) “Le principe de responsabilité” publié peu de temps avant, en 1979, où l'auteur conditionne la possibilité de vie des générations futures à l'engagement des générations actuelles.

Mais cet engagement ne peut et ne doit certainement pas faire l'économie de ces repères catastrophiques car, ils sont, individuellement et dans leur ensemble, l'expression des dogmes historiques fondant la modernité qui leur a 
donné naissance. Nous n’interrogeons pas ici les héritiers plus ou moins lointains du Siècle des Lumières, de la Raison et de la Révolution française, le capitalisme ou le marxisme qui sont toujours présents en toile de fond. Il semble, en effet, plus important de nous tenir à la considération des relations sociales comme des institutions au sens où elles sont toujours la résultante de la définition que les hommes se donnent de leur propre humanité, de leur imaginaire social et de leur conception du monde. En effet, la lecture du social, par le biais de l'institution, ne le fige ou ne le pérennise pas car le social est justement le lieu de rapprochement où les institutions, comme formes particulières, sont mises en concurrence et tendent à légitimer, par la contrainte, certaines formes de domination plutôt que d'autres.

Actuellement, la domination exercée est celle de la science et de la technique. Cette domination s'appuie particulièrement sur le fantasme de la maîtrise de la nature par l'homme. Mais face à des catastrophes comme celles évoquées, l'exercice de cette domination provoque des contestations et de résistances qui sont autant de symptômes d'une prise en compte de la menace qu'elle représente.

Ces limites sont repérables dans la sociopathologie car elle impose de se dégager des carcans évolutionnistes et historicistes pour mettre en évidence des caractéristiques anthropologiques qui, bien que favorisant la domination du couple science et technique ne le font plus avec autant de succès que par le passé. Et cela est particulièrement visible dans le domaine sanitaire où les Etats démocratiques se sont dotés d'appareils dit de "Santé publique" pour concilier des positions et des intérêts contradictoires. L'approche par la sociopathologie s'inscrit dans une approche socioanthropologique des sociétés visant à modifier l'appréhension classique de la modernité qui est caractérisée par une lecture souvent évolutionniste. Et il est nécessaire, de ce point de vue, de faire un détour pour repositionner les éléments de la discussion et cela particulièrement, ici, pour les idéologies sédimentées sous une forme institutionnelle, comme la santé publique, qui conditionne les situations du "vivre la catastrophe”.

\section{De la limite de la Santé publique}

La Santé publique est généralement dominée par une volonté politique de prévention où elle se laisse "définir comme un substrat culturel, c'est-à-dire propre à faire adhérer un maximum d'acteurs sociaux - depuis les citoyens ou usagers jusqu'aux pouvoirs publics en passant par les corps et les institutions 
intermédiaires - à ses prescriptions de réduction des risques individuels et collectifs”. (Dozon, 2001, p. 25). La Santé publique peut aussi s'exprimer comme le propose D. Fassin (2001, p. 53):

Au risque de paraphraser son nom, on dira que la santé publique se caractérise par la croyance, obligatoirement partagée parmi ceux qui s'en réclament, en une valeur commune: la santé comme bien public. Autrement dit, elle pose, d'une part, la santé comme un bien supérieur - ce qui ne signifie pas qu'il y ait accord sur ce qu'est la santé - et, d'autre par, la santé comme un bien public - ce qui n'implique pas non plus un consensus sur ce que veut dire l'adjectif public.

Avec Fassin, la Santé publique se situe au niveau de la croyance, comme un élément culturel définissant la société. La santé est considérée alors comme un principe important, privilégié socialement comme une valeur en soi. La Santé publique est donc dans une relation concurrentielle avec d'autres éléments culturels et cela d'autant plus que son appartenance à ce qui pourrait ressortir d'un bien public n'est pas acceptée par l'ensemble de la société.

Mettre en perspective les catastrophes, par l'approche de la Santé publique ou par divers aspects législatifs, présuppose un relativisme culturel immédiat. C'est ce qui nous amène à privilégier l'usage de la sociopathologie pour saisir, non une définition culturelle d'une maladie mais, le développement d'une vulnérabilité adossée à un accroissement d'une crise du symbolique dans une période de généralisation de menaces, face à des risques technologiques et environnementaux d'une part, et socio-économiques d'autre part. Cette généralisation des menaces est liée au développement techno-scientifique qui accompagne ou provoque les mutations sociales visibles particulièrement dans les modes de vie des habitants des pays riches où toutes les couches de la société sont exposées à la menace de catastrophe écologique. Il ne s'agit pas seulement des risques technologiques majeurs mais aussi des menaces qui, pour être diffuses, n'en sont pas moins inquiétantes. Les risques concernent tout le monde comme Tchernobyl le montre. Les frontières sont obsolètes tant dans l'espace que dans le temps. Pourtant l'usage de la conceptualisation des pathologies perdure grâce à une enveloppe culturelle alors que le risque est tout autant social et économique que sanitaire. ${ }^{3} \mathrm{D}$ 'ailleurs, une des caractéristiques

${ }^{3}$ Le même travail peut être fait avec la production d’Organismes Génétiquement Modifiés tant ils catalysent des incertitudes augmentant un sentiment de vulnérabilité hors de toute considération ordinaire.

Horizontes Antropológicos, Porto Alegre, ano 12, n. 25, p. 191-216, jan./jun. 2006 
de cette incertitude nouvelle est qu'elle engendre une vulnérabilité radicale qui ne peut être limitée tant elle met à mal les bases sociales et anthropologiques qui permettaient la prise en charge traditionnelle des incertitudes et tout particulièrement celles liées à la maladie. La vulnérabilité croissante expose donc les populations à une fragilité d'autant plus grande qu'elle renvoie à la disparition des options de préservation puisées ordinairement dans les registres tant matériels que culturels ou symboliques.

D'ailleurs, si on analyse les risques techno-scientifiques du point de vue de la vulnérabilité, la population apparaît exposée à une perte de repères structurés sur l'inscription sociale et le caractère collectif de la santé publique ne semble pas être en mesure de prendre en compte ce déficit. La vulnérabilité et son corollaire, l'incertitude progressent et participent alors en tant qu'accélérateurs d'inadaptation dans une dynamique triangulée formée des acteurs eux-mêmes, des institutions et des pathologies. C'est par cette mise en cohérence qu'il est possible d'analyser la dimension sociopathologique comme un indicateur du développement de l'incertitude et de la vulnérabilité dans les situations de crises et/ou de catastrophes car l'une des caractéristiques plus abstraites des sociopathologies est, selon nous, qu'elles apparaissent dans un contexte de désajustement de certaines institutions entre-elles.

\section{De l'intérêt de la sociopathologie}

Le concept de sociopathologie renvoie à un registre explicatif triple. Le premier élément constitutif de la sociopathologie renvoie à la gestion des risques, à la réduction de la vulnérabilité et de l'incertitude qui l'accompagne. Cette dimension intègre des réponses élaborées par des technocrates et les gestionnaires des risques présentant, à la population, des compétences en terme de maîtrise de l'évènement ou, du moins, des garanties quant à leur propre capacité à effectuer un "retour d'expérience". Le message alors adressé à la population, par ces professionnels, se veut le plus souvent rassurant quant à la qualité de leurs interventions toujours présentées comme des gages définitifs de la suprématie de la science et de la technique face à l'imprévu et à l'insaisissable. Le deuxième élément correspond au recours aux ressources traditionnelles de la culture pour atténuer l'indétermination et reconstruire une espérance. Mais l'efficacité de ces ressources est contestée par Isabelle Rieusset-Lemarié (1992). En effet, elle considère que la culture classique ne 
peut endiguer ce qui relèverait de phénomènes épidémiques modernes. Selon elle, pour se protéger, la société doit de se doter d'une culture nouvelle car la tradition atteindrait ses limites au contact des menaces du monde moderne.

Ces premiers éléments succinctement évoqués présentent des différences de nature tout en portant chacun une argumentation cohérente qui permet d'appréhender le risque et la catastrophe; pourtant ils sont insuffisants pour concevoir la dimension sanitaire et sociale induite en situation d'urgence. Car, précise Claudine Herzlich (1991):

La maladie, une des expériences humaines les plus importantes, représente une articulation très étroite de ce qui est le plus individuel et le plus collectif. C'est un phénomène éminemment social parce que chaque société développe des représentations très spécifiques et que les maladies ont un impact sur le développement économique des sociétés. C’est ce que montre les grandes épidémies du Moyen Age ou le retour d'une épidémie comme le sida. Pour y faire face, toutes les sociétés doivent développer des systèmes de réponse et de prise en charge.

Partant de cette analyse, il faut considérer comme une institution les réponses formulées par les gestionnaires des risques face à celles de la tradition. Elles attestent du caractère moderne du troisième élément de la sociopathologie apparaissant dans ou par l'influence des sociétés à développement technicoscientifiques (Beck, 1987, 2001) où la pathologie émerge comme signe de la transformation des sociétés. Son examen permet de faire état de la mise en cause de formes sociales évaluées comme caduques au profit de formes sociales émergeantes intimement liées au caractère techno-scientifique du développement des sociétés. Cette transformation dans l'appréciation des pathologies n'est, quant à elle, pas un phénomène récent car Ivan Illich (1975) dans "émésis médicale”, voyait déjà disparaître la part de liberté de l’individu derrière la contrainte d'une croyance en la science et la technique au profit de l'émergence d'une forme de symbolisation nouvelle ancrée à la raison et à la professionnalisation médicale.

D'ailleurs et c'est principalement ce que pressant Illich, ce sont les nouvelles orientations médicales et le redéploiement des pratiques déjà connues qu'il s’agit de considérer pour aborder les catastrophes. En effet, c'est en dépassant la dimension physiologique (qui est par ailleurs socialement construite), en 
interrogeant les rôles de la science et du praticien qui la sert; ${ }^{4}$ ou encore celui des institutions pour lesquelles ce dernier intervient professionnellement qu'il est possible de saisir l'intérêt global de la sociopathologie. La sociopathologie est, comme les appareils institutionnels qui retiennent notre attention, à tous les niveaux de la société, dans le plus local et particulier, comme dans le plus global et général. D’ailleurs, c'est dans toutes les dimensions des rapports sociaux constitués de pratiques et d'usages, de domination et de contrainte que nous repérons des éléments de la sociopathologie. Il va alors de soi, faut-il le préciser, que l'hégémonie des disciplines médicales est analysée comme un rapport de domination dans un système organisé car, comme le souligne Herzlich (1991):

Un système de santé est efficace non seulement en fonction des thérapeutiques médicales disponibles, mais aussi de ses modalités d'organisation et des conceptions qui président aux grandes priorités qu'il se donne "ou encore" l'état de santé des populations dépend autant des facteurs économiques et socioculturels que des progrès médicaux.

D’ailleurs, nous nous permettrons de compléter sa belle formule "On doit avoir une conception intégrée de la santé qui part de la molécule pour arriver à la société” (Herzlich, 1991) par: on doit aussi avoir une conception intégrée de la santé qui parte de la société pour arriver à la molécule, tout en considérant les ruptures dans le continuum. Et c'est en suivant cette logique que nous devons analyser le rôle joué par l’OMS dans la gestion des conséquences de Tchernobyl.

\section{L'OMS à l'épreuve des faits}

S’il est une pathologie souvent convoquée dans le cadre des conséquences de Tchernobyl, c'est la radiophobie. La radiophobie illustre parfaitement le rôle joué, sur le terrain de la vie quotidienne, par les appareils institutionnels.

La vocation de l'OMS est clairement explicitée dans le seul article de son chapitre I: “il s’agit d'amener tous les peuples au niveau de santé le plus élevé

\footnotetext{
4 A titre d'exemple, nous pouvons évoquer la situation dans laquelle se trouvent certains chercheurs. En Bélarus Bandajevsky, a été maintenu en détention avec, à sa sortie, une interdiction de diriger des travaux salués par des instances scientifiques internationales ou, en France, Viel, pris dans une polémique où il a été très sévèrement et publiquement critiqué quant à ses compétences.
}

Horizontes Antropológicos, Porto Alegre, ano 12, n. 25, p. 191-216, jan./jun. 2006 
possible” (OMS, [s.d.]). Dans le préambule de sa constitution, il est précisé que "La santé est un état de complet de bien-être physique, mental et social, et ne consiste pas seulement en une absence de maladie ou d'infirmité”. Il y est souligné que "la possession du meilleur état de santé possible" est un droit fondamental de tout être humain "quelles que soient sa race, sa religion, ses opinions politiques, sa condition économique ou sociale”. Structure administrative supra nationale, l'OMS ne peut intervenir auprès des populations sans accord des Etats concernés car "Les gouvernements ont la responsabilité de la santé de leurs peuples; ils ne peuvent y faire face qu'en prenant les mesures sanitaires et sociales appropriées”.A l'instar de l'ONU dont elle est une constituante, l'OMS s'organise autour de principes politiques. Mais, elle est parfois dénoncée pour des alliances institutionnelles qui donnent à son action des orientations contestées au regard de sa genèse. Ainsi en irait-il, selon Michel. Fernex (2001, p. 246-247), d'un accord signé en 1959, avec l'AIEA qui restreint l'activité de l'OMS. Fernex souligne particulièrement l'article III de l'accord qui propose des "arrangements qui pourraient être nécessaires pour sauvegarder le caractère confidentiel de certains documents" et le met en résonance avec le point 3 de l'article I de ce même accord:

Chaque fois qu'une des parties se propose d'entreprendre un programme ou une activité dans un domaine qui présente ou peut présenter un intérêt majeur pour l'autre partie, la première consulte la seconde en vue de régler la question d'un commun accord.

Les intérêts de l’AIEA, centrés sur le développement de l'énergie atomique, peuvent donc dans le cadre de l'élaboration d'un "commun accord” être supérieurs et invalider la mise en place d'études risquant de leur nuire à l'industrie nucléaire. On peut y voir les raisons qui aboutissent à l'intérêt fort relatif de l'OMS pour l'analyse des conséquences de Tchernobyl. C'est assurément l'avis de Yves Lenoir qui a analysé les liens structurels unissant l'AIEA et l'OMS, et montré que la promotion de l'énergie atomique constituerait l'une des missions officielles de l'OMS:

La promotion simultanée de la santé publique et d'une activité industrielle à risque élevé pour la santé fait donc partie des objectifs statutaires de l'OMS, sans que personne ne s'en soit jamais ému ni, a fortiori, qu'une telle aberration ait provoqué le moindre conflit apparent au sein de l'organisation. (Lenoir, 1996, p. 14). 
Selon ses statuts, la vocation de l'OMS est d'intervenir à Tchernobyl avec entre autres missions, l'évaluation des effets sanitaires de la diffusion des radionucléides dans l'environnement afin de mettre en place les moyens appropriés pour protéger les populations. Rappelons les effectifs concernés actuellement: environ 2 millions de personnes sont exposées aux radionucléides. Parmi elles, l'estimation de 500.000 enfants qui vivent dans les zones fortement contaminées n'est plus contestée. Il faut aussi comptabiliser les personnes relogées qui ont été évacués et les quelques 800.000 liquidateurs dispersés ${ }^{5}$ sur l'ensemble des républiques de l'ancienne URSS. Mais selon l'accord déjà évoqué, la nature industrielle de la centrale de Tchernobyl imposa, dès les premiers instants, l'avis de l'AIEA qui est aussi liée à l'ONU. Face à un évènement spécifique, deux appareils spécialisés intervenant chacun sur son champ d'expertise. L'esprit de l'ONU qui préside aux destinées, tant de l'OMS que de l'AIEA, incline à considérer que la supériorité de l'intérêt pour la santé des populations est indiscutable et subordonne automatiquement l'AIEA à l'OMS qui reçoit alors aide et soutien, tant humain que technologique, particulièrement par la mise à disposition de spécialistes et de scientifiques de l'atome, qu'en fournissant les retours d'expériences acquis lors des accidents nucléaires antérieurs. Mais de l'esprit à la lettre, il y a parfois des écarts importants et c’est certainement l'accord de 1959 qui renversa la prépondérance humanitaire au profit de l'industriel dans la logique soulignée par Claude Lefort (1981, p. 189): “L’expérience prouve que les gros contrats se négocient fort bien entre les puissances ouvertement adverses [...]”. Cette domination de l'AIEA sur l'OMS est d'ailleurs aussi tout à fait conforme au rapport technique que cette dernière publie, en 1958, sous le titre "La politique à suivre en cas d'accident”:

Cependant, du point de vue de la santé mentale, la solution la plus satisfaisante pour l'avenir des utilisations pacifiques de l'énergie atomique serait de voir monter une nouvelle génération qui aurait appris à s'accommoder de l'ignorance et de l'incertitude.

Comme Fernex (2001) le souligne “Cette apologie de l'ignorance reflète un mépris des populations, contraire à l'esprit et à la lettre de la Constitution de

5 Il ne s'agit que d'évaluation mais un accord se dessine au niveau de 800.000 individus. 
l’OMS”. Pourtant, toujours selon lui, ce passage a été lu à la Conférence organisée par l'OMS sur Tchernobyl, en novembre 1995, à Genève. L'orateur a également cité un Directeur Général de l'AIEA, qui a déclaré le 28 août 1986, qu'au regard de "l'importance de l'énergie atomique, le monde pourrait supporter un accident comparable à celui de Tchernobyl par an” avant de conclure sur une position allant dans le sens de l'esprit et de la lettre de l'OMS: "Un Tchernobyl suffit. Il faut tendre vers une sécurité totale”. ${ }^{6}$ Le vingtième anniversaire de Tchernobyl arrivera bientôt, espérons-le, sans que la régularité statistique de ce type d'évènement puisse être vérifiée. Mais, même si c'est le cas, si une catastrophe nucléaire se produit de nouveau, l'OMS respecte sa signature où elle cherche le "commun accord" avec l'AIEA, où elle fait, partiellement, mais nécessairement sienne la vocation de l'AIEA dont l'un des articles fondateurs précise que sa vocation est “d'accélérer et d'accroître la contribution de l'énergie atomique pour la paix, la santé et la prospérité dans le monde entier". ${ }^{7}$ En parfaite réciprocité, l'OMS et l'AIEA doivent éviter de mener des recherches dont les résultats gêneraient l'autre. Contractuellement, les signataires indiquent qu'ils seront appelés à prendre des mesures de sauvegarde pour garantir la confidentialité de renseignements tout en s'informant réciproquement quant aux projets et programmes prévus. La confidentialité requise est une forme objective de censure à l'égard du public qui est destinataire en dernière instance du travail de l'OMS sauf à considérer que les objectifs initiaux exprimés au Chapitre I de sa Constitution "d'amener tous les peuples au niveau de santé le plus élevé possible.” puissent être atteints sans en informer les populations.

Pourtant, en son chapitre II sont indiqués les moyens dont doit se doter l’OMS pour atteindre son objectif. L'Organisation se doit d'agir comme autorité directrice et autorité coordinatrice dans le domaine de la santé; elle se doit de

\footnotetext{
6 Le conditionnel, que nous utilisons comme Fernex, est imposé car les actes de cette conférence de 1995 ne sont toujours pas publiés: “Cette intervention, comme tant d'autres présentées à la Conférence de l’OMS, à Genève, devait être publiée dans les actes: un document très attendu, qui avait été promis par le secrétariat de la Conférence pour mars 1996. Pourtant ces actes n’ont pas encore paru en ce début du XXIe siècle. Certains pensent que ces textes risquaient de profondément perturber la Conférence de l'AIEA à Vienne, prévue pour avril 1996. On pense que les manuscrits ont été enterrés, voire censurés, grâce à l'Accord avec l'AIEA, signé par l’OMS en 1959" (2001, p. 246).

${ }^{7}$ Cette phrase tirée des statuts de l'AIEA, figure sur les premières pages des publications de cette Agence, y compris dans les actes de la conférence de l'AIEA d'avril 1996 sur Tchernobyl.
}

Horizontes Antropológicos, Porto Alegre, ano 12, n. 25, p. 191-216, jan./jun. 2006 
fournir l'assistance technique appropriée et, dans les cas d'urgence, l'aide nécessaire, à la requête des gouvernements ou sur leur acceptation; elle doit fournir toutes informations et donner tous conseils et toute assistance dans le domaine de la santé; et aussi, elle doit aider à former, parmi les peuples, une opinion publique éclairée en ce qui concerne la santé. Il semble que ce soit des objectifs difficiles à atteindre sans prendre en considération la population et l’information minimale qui lui serait alors dédiée.

Si le recours à l'usage du nucléaire dans la pratique industrielle est présenté comme une fatalité et un destin technologique incontournable, l'OMS confrontée à "un Tchernobyl par an” peut travailler comme elle le fait actuellement à maintenir le niveau de santé le plus élevé possible. Mais alors, le nuage de Tchernobyl n'a pas seulement contaminé l'environnement et les corps, il a aussi assombri les esprits, la liberté individuelle et collective car, dans la situation provoquée par l'émergence de l'atome artificiel dans la vie quotidienne, seuls, les experts délégués par l'AIEA et l'OMS disposent des informations et de l'autorité nécessaire pour définir les formes de la réalité quotidienne et d'une vie normale. Certaines autorités nationales peuvent d'ailleurs d'une certaine manière être aussi privées d'informations utiles à la sécurité des populations si elles ne peuvent élaborer de manière indépendante des mesures environnementales. Et de ce point de vue, l'accord évoqué contraint l'OMS au passage sous les fourches Caudines de l'AIEA donc l'OMS perd son autonomie quant à ses orientations de recherche et les publications de résultats. Dans le cas qui nous occupe, l'OMS est dans l'incapacité objective de construire des informations pour assurer aux populations la mise en place de mesures de prévention face aux effets pathogènes de la radioactivité artificielle de Tchernobyl. Et contre son propre projet éthique, l'OMS ne peut participer à la formation d'une opinion publique éclairée par toutes les lumières mais seulement par les rais autorisés par l'industrie atomique et sa représentation, l'AIEA. De manière définitive, en paraphant l'accord évoqué, lors de la $12^{\mathrm{e}}$ Assemblée Mondiale de la Santé, le 28 mai 1959, l'OMS s'est aliéné à l'AIEA et cela ne s'est pas fait sans opposition mais assurément contre l'avis d'un groupe d'experts dans le domaine de la génétique, dont le titulaire du Prix Nobel, J.-M. Muller, qui réunis à Genève en 1956 considèrent que:

Le patrimoine génétique est le bien le plus précieux de l'être humain. Il détermine la vie de notre descendance, le développement sain et harmonieux des générations 
futures. En tant qu'experts, nous affirmons que la santé des générations futures est menacée par le développement croissant de l'industrie atomique et des sources de rayonnements. Nous estimons également que les mutations nouvelles qui apparaissent chez les êtres humains, seront néfastes pour eux et pour leur descendance. (OMS, 1957, p. 183).

Du point de vue sociologique, cet accord relève de la mise en réseau de l'OMS et de l'AIEA qui s'organisent autour d'une même vision du monde. En toute logique, l’OMS devait éviter tout accord de réciprocité et de confidentialité entraînant une forme de mise sous le boisseau de ses objectifs initiaux, et cela d'autant que l'appareil partenaire développe des activités sociopathogènes. Quoiqu'il en soit, les travaux de l'OMS sont toujours concentrés sur les expositions massives à la radioactivité en excluant l'incorporation des faibles doses. C'est une attitude que des scientifiques et contestataires dénoncent: “On est, dans le cas de Tchernobyl, en présence d'un effet de dilution des doses d'irradiation et on ne sait rien ou presque sur l'effet cancérigène de très faibles doses” (Belpomme, 2004, p. 56). Nous l'avons évoqué, l'information ne peut être totale car elle est structurée par les décisions prises lors des conférences internationales organisées par une OMS inféodée à l'AIEA. Mais les autorités nationales responsables, selon l'OMS, de la mise en œuvre des mesures de protection de la population ne peuvent alors bénéficier, par autocensure, de la formation nécessaire à la prévention. D’ailleurs c'est l'AIEA qui se pose en interlocuteur privilégié pour répondre à l'émoi suscité par la catastrophe de Tchernobyl au niveau international et qui investit le champ sanitaire laissé vacant par l'OMS.

En effet, l'AIEA organise une conférence internationale "Une décennie après Tchernobyl” où les maladies, en dehors de celles liées aux expositions directes aux radiations ionisantes, sont rejetées. Cette labellisation provoque le rejet massif de demandes de prises en compte de maladie, soit dans un registre psychologique (où sont répertoriées les pathologies diagnostiquées comme relevant de montées d'angoisse infondées), soit dans un registre social (de stratégies d'acteurs considérés opportunistes et usurpateurs, fraudeurs ou simulateurs dont l'unique objectif serait l'acquisition d'avantages spécifiques).

En définitive, la conférence reconnaît seulement le "syndrome d'irradiation aiguë” comme une “conséquence de l'accident” et l'existence de troubles neuropsychiatriques chez des enfants dont les mères ont été irradiées pendant leur grossesse. Toute hypothèse liant la survenue de troubles neuropsychiatriques 
chez les adultes à leur exposition aux radionucléides est rejetée. Cette absence de relation causale ne correspond pas seulement à un choix d'orientation thérapeutique, elle permet aussi en usant d'un processus d'identification, de réduire considérablement les effectifs des victimes. Et ceux qui n’entrent pas dans les catégories déterminées par les autorités ne peuvent prétendre à des aides spécifiques.

La logique théorique qui préside à la mise en forme de la nomenclature des pathologies peut se résumer ainsi: il existe bien des pathologies physiologiques consécutives à l'exposition aux radionucléides diffusés dans l'environnement par l'explosion de Tchernobyl et la liste est établie par l'OMS. La connaissance et la conscience de la catastrophe provoquent des pathologies d'ordre psychologique ne pouvant être attribuées de manière directe à l'explosion. Elles ne seront donc pas prises en compte dans l'évaluation des conséquences sanitaires de Tchernobyl. D'une certaine manière, il ne s'agit de rien de moins que d'une individualisation d'un phénomène collectif. où chacun se doit de gérer l'évolution de sa santé en fonction de la catégorie auquel il appartient et de ce qu'il obtient comme renseignement sur la situation. Et là, la notion d'information est de première importance.

\section{L'absence d'information comme garantie du silence}

Le rôle de l'information est déterminant lorsqu'il s'agit d'aborder les catastrophes. Certains auteurs proposent de suivre des stratégies de "bouclage des médias" (Lagadec, 1981, p. 258-259) dans le cadre des consignes de gestion de crises. Avec Tchernobyl, nous sommes, certainement et plus fermement, dans une forme de bouclage de l'opinion visant à organiser l'opinion dans la perspective de la survenue inéluctable de crises ou le procès de colonisation du futur proposé par Anders (2002).

A défaut de bouclage préventif de l'opinion au moment de Tchernobyl, il a fallu “boucler" l'appareil industriel et politique pour que rien n'en sorte. Pour boucler l'opinion française, l'Etat a laissé a délégué, à ses appareils technocratiques, le soin de développer un discours scientifique auto légitimant; pour l'opinion soviétique, les usages politiques coercitifs du moment se sont presque suffi à eux-mêmes. Cependant, la volonté de maîtrise a exigé l'usage de quelques artifices pour permettre aux autorités de gérer les populations les plus exposées. Il a fallu identifier le phénomène car les premières évacuations 
se sont déroulées en l'absence d'information sur les motivations de ce transfert massif de population. Les déplacements sont d'abord provisoires, "pour quelques jours”. Rien n'est dit sur la situation. Les riverains vaquent d'ailleurs souvent à leurs occupations habituelles avant qu'ils ne reçoivent l'ordre d'évacuer. Les premiers silences des autorités soviétiques ont été prolongés, officiellement pour ne pas accabler les populations et permettre leur meilleure prise en charge. L'information est secrète comme si la connaissance de l'état de l'environnement était pathogène et qu'il s'agissait de la dissimuler pour protéger les individus directement exposés mais aussi d'une certaine manière, de l'humanité; comme si la réalité de l'explosion était une arme qu'il fallait désactiver avant qu'elle ne tombe aux mains d'opposants. ${ }^{8}$ D'ailleurs le message diffusé en France est clair, les conséquences de Tchernobyl ne sont en aucun cas pathogènes et elles n'ont de sens que parce que leurs inventeurs, les mouvements écologistes, peinent à obtenir du crédit politique sans elles. Logiquement alors, les pathologies attribuées à Tchernobyl seraient inventées par les écologistes pour développer leur surface d'influence en provoquant, chez les individus, une peur de l'environnement utilisée à des fins politiques.

Mais le modèle gestionnaire utilisant le silence est dépassé face à une crise majeure comme Tchernobyl. En effet, le risque industriel a changé de nature et d'échelle. La notion d'accident technologique, qui est le propre de notre société moderne, a en s'imposant épuré les analyses de tout fatalisme et la rationalité expérimentale a mis l'homme face à certaines responsabilités. Ainsi, l'accident technologique majeur n'est pas limité et atteint les contrées les plus éloignées et les plus étrangères au phénomène en y modifiant les situations sanitaire, écologique et sociale. ${ }^{9}$ La connaissance de l'explosion de Tchernobyl n'est plus confidentielle. Les populations parfois tardivement en sont informées et les promoteurs du nucléaire recourent alors à un concept fondamental, la radiophobie, pour expliquer les effets sanitaires de l'explosion. Avec dextérité et un usage paré de garanties scientifiques auto administrées, les experts

${ }^{8}$ En 1957, à Kyshtyn-Tchéliabinsk, un centre de déchet radioactif a explosé. Le nuage contaminé n'est pas sorti des frontières de l'URSS. Le système soviétique s'est tu sur l'événement. Mais aussi la CIA qui avait détecté le nuage radioactif.

${ }^{9}$ Loin de la centrale, le passage du nuage de Tchernobyl a modifié la culture des Lapons: il a été procédé à l'abattage de troupeaux entiers de rennes et des interdictions de pratiques de la pèche qui sont deux éléments essentiels de la culture pastorale.

Horizontes Antropológicos, Porto Alegre, ano 12, n. 25, p. 191-216, jan./jun. 2006 
expliquent l'importance qu'il y a à prendre en compte la dimension psychologique induite par l'évènement:

Les différentes mesures pour protéger la population n’ont pas toutes la même importance, et cela s'applique également sur l'effet psychologique défavorable qu'elles peuvent provoquer, de ce point de vue, l'évacuation de la population est l'action de loin la plus complexe que l'on puisse avoir à prendre. [...] Il s'ensuit que la sélection des niveaux de danger impliquant l'introduction de telle ou telle mesure de protection ne doit pas être seulement basée sur des considérations quant au risque biologique d'une exposition aux radiations, mais aussi sur l'effet psychologique négatif et la nocivité pour la santé publique ${ }^{10}$ qui pourraient résulter de la mise en œuvre d'une mesure donnée. (Bulletin AIEA, 1987).

Ce rapport de l'AIEA précise aussi que l'analyse comparée de groupes d'individus vivant sur les zones contaminées ne présente pas de "déviations" notables par rapport à un groupe de contrôle non exposé à la radioactivité. En clair, être exposé à la radioactivité ou pas, entraînerait peu de différences, des écarts non significatifs, hors des hautes contaminations. Les seules différences notables indiquées par l'AIEA sont l'inquiétude développée par les personnes vivant en zones contaminées pour la santé de leurs enfants, la faiblesse qu'elles ressentent et la mauvaise appréciation de leur santé. Nous sommes loin d'une situation normande. Mais portant jusqu'au bout sa logique qui souhaite "voir monter une nouvelle génération qui aurait appris à s'accommoder de l'ignorance et de l'incertitude" (AIEA, 1958), l'AIEA conclut son bulletin en considérant que:

Cette tension et cet état de stress chronique sont la cause du syndrome de radiophobie affectant une partie de la population; dans la situation actuelle de radiation elles peuvent même faire peser une menace sur la santé plus grande que l'exposition aux radiations elles-mêmes.

La principale menace serait une tension psychologique provoquée par l'effort d'adaptation, la radiophobie, à ce que l'individu perçoit comme un environnement menaçant. La permanence de cette activité d'ajustement psychologique, ce stress chronique serait une cause plus importante de pathologie

${ }^{10}$ C'est nous qui soulignons. 
que l'exposition physiologique aux radiations. Logiquement, c'est bien l'information qui devient pathogène et même sociopathogène car en présentant aux habitants leur environnement comme étant dangereux, elle provoque des effets de stress. Pourtant, concernant la radiophobie, nous pouvons préciser que jamais, lors toutes nos recherches sur les territoires officiellement contaminés, nous n'avons rencontré d'individu se déclarant atteint par le syndrome de la radiophobie. Une femme, hospitalisée dans une ville ceinturée par des zones officiellement très fortement contaminées, s’est exprimée au sujet de la radiophobie. En s'esclaffant devant une chambrée nombreuse qui acquiesça, elle nous déclara que si la radiophobie existait, ceux qui en ont été atteints sont morts depuis longtemps car il est impossible de vivre sans manger. La radiophobie résultant d'une hantise par rapport à la contamination de l'environnement comme seule explication au développement des pathologies parait pour le moins irréaliste et ce dernier propos de sens commun semble plus rigoureux, pour la description des conséquences psychologiques de l'évènement Tchernobyl, que les conclusions et concepts de nombreux experts ou scientifiques convoqués tant par l'OMS que l'AIEA.

En effet, les personnes qui se déclarent atteintes par les retombées de Tchernobyl constatent des symptômes, désignent des manifestations et surtout identifient les causes des maux qui les affectent. Nous sommes proche des cas observés et analysés par Marcel Mauss (1926) car la radiophobie ou le stress environnemental ne permettent pas de trouver, dans l'ordre de la suggestion collective, une explication aux pathologies qui leur sont imputées car, nous dit Mauss, il nous faudrait être face à une situation présentant des modalités particulières qu'il définit dans ces termes:

Cet état coïncide généralement avec une rupture de communion, soit par magie, soit par péché, avec les puissances et choses sacrées dont la présence, normalement le soutient. La conscience, est alors tout entière envahie par des idées et des sentiments, qui sont entièrement d'origine collective, qui ne trahissent aucun trouble physique. L'analyse n'arrive à saisir aucun élément de volonté, de choix, ou même idéation volontaire de la part du patient, ou même de trouble mental individuel, hors de la suggestion collective elle-même. Cet individu se croit enchanté ou se croit en faute et meurt pour cette raison. (Mauss, 1926).

Les malades rencontrés, tant individuellement que collectivement, ne se considèrent pas en faute mais victimes. Lorsque la notion de faute est évoquée, 
elle est attribuée aux responsables soviétiques ou à leurs cautions actuelles, mais jamais endossée par ceux qui sont atteints dans leur chair. Ces derniers alimentent tout autrement le registre de la sociopathologie de Tchernobyl que ne le font les agents des appareils chargés de la gestion des conséquences de Tchernobyl qui sont aussi, dans le même temps, porteurs d'un discours idéal auto légitimé.

Après ce large détour indispensable pour apprécier le rôle et l'activité d'appareils institutionnels comme l'OMS et l'AIEA, et avant de conclure, nous voulons proposer un long extrait d'entretien que nous ne découpons pas pour souligner combien les situations sociales sont complexes en situation de catastrophe. Cet extrait représente aussi ceux auxquels nous n'avons pas, dans cet article, donné la parole, les habitants des zones contaminées et il illustre bien l'importance qu'il y a à considérer les pathologies non seulement comme une situation mais bien comme un processus social agissant dans toutes ces facettes de la vie quotidienne où se projettent les cadres sociaux institués par les autorités nationales et internationales que nous venons de présenter. Ces logiques institutionnelles sont bien présentes dans le rapport à la pollution radioactive environnementale et, de ce fait, sont autant de pathologies sociales latentes qui deviennent sociopathologies vécues comme personnelles du fait de leur incorporation. Et ces pathologies peuvent être alors considérées comme des institutions en train de naître.

Une des illustrations les plus caractéristiques de ce phénomène d’incorporation institutionnelle concerne une femme qui n’a jamais été évacuée. Une représentante des évacuations ratées en quelque sorte. Cette femme est une kolkhozienne de 60 ans, retraitée. Elle habite dans un village en zone officiellement très contaminée où le relogement est théoriquement obligatoire. Elle demeure en ces lieux où les autorités considèrent qu'il n’y a personne car il est interdit d'être là. Elle vit dans le village où elle est née et travaillée à la traite des vaches. Elle connaît l'obligation de relogement mais déclare qu'elle ne peut plus partir car elle s'est habituée à la radioactivité. Son affirmation contredit l'hypothèse selon laquelle la vie dans un environnement contaminé est un facteur pathogène. Mais son avis est fondé sur les conseils de médecins consultés hors de la zone contaminée. Ceux-ci considèrent qu'elle est entrée dans un rapport de dépendance à la contamination. Cette kolkhozienne serait addicte aux radionucléides. La situation est paradoxale au regard du niveau élevé de radiations qui a provoqué l'évacuation de la population locale. Pour elle, l'exposition permanente à la radioactivité environnementale serait une 
situation indispensable pour qu'elle puisse poursuivre une vie normale. Et là, les médecins, comme membres de l'appareil sanitaire national, participent pleinement à la construction sociopathologique de Tchernobyl et cela d'autant plus qu'ils légitiment objectivement une situation à risques élevés. Mais pour cette kolkhozienne la situation est complexe. Elle exprime parfaitement comment des formes de résistances sociales surgissent face aux positions imposées par les appareils (Etat, AIEA, OMS, ...).

Dans l'encart qui suit, nul trait de détresse psychique, ni de comportement déviant. Seulement l'expression d'une vulnérabilité face à l'indétermination que la société, en situation post-catastrophique, ne semble pas pouvoir résoudre.

Ici, la radioactivité est importante. Mais on vit, on n'a pas peur. Personne ne nous dit le niveau de contamination. Ils viennent, ils prennent des mesures et ils repartent. On ne connaît pas le montant des indemnités auxquelles ont droit les habitants des zones contaminées car on ne les touche pas. Nous n'avons pas d'indemnité et la retraite ne nous suffit pas. Elle ne nous suffit même pas pour se nourrir le mois entier. Je ne peux pas partir et pour aller où? Qui va me donner un logement? Qui va m'aider? Ici, je m'aide moi-même car j'ai une vache, un cochon et je cultive les pommes de terres, les tomates, les concombres. Je peux déjà manger... Sinon, il faut aller au magasin, mais je ne peux pas, j'e n'ai pas d'argent pour ça [...]. Notre village devait être relogé. Mais ils tardaient, après ils ont dit: vous pouvez rester! Et on est resté. Et tous les natifs de ce village ne voulaient pas partir [...]. Il n’y que 5 familles qui sont parties. Et ceux qui sont partis, ils auraient été ravis de revenir, mais il n’y a plus de logement libre. Leurs maisons sont déjà occupées par d'autres gens. Mais s'ils veulent revenir, c'est pour avoir leur potager [...].Personne ne veut plus partir car ici, on peut cultiver et avoir des animaux. Personne ne veut partir, parce qu'on ne sait pas ce qui nous attend. Làbas, il n'y a pas de travail et au moins ici, on nous verse nos retraites chaque mois. C'est pas grand chose, mais on a de quoi s'acheter du pain [...]. Alors, je suis née ici, baptisée ici, j’ai passé toute ma vie ici, et je vais mourir ici [... . . Le 27 avril 1986 [Le lendemain de l'explosion de Tchernobyl], je vivais dans la maison de mon frère. On a remarqué que les feuilles sont devenues jaunes. Les feuilles ont jauni à moitié: un côté jaune, l'autre vert. Du côté de passage de radiation, elles étaient jaunes... Ici, la radioactivité était très importante. Depuis, rien n’a changé. On vit peu à peu: S'il n'y a pas de pain aujourd'hui, il y en aura peut-être demain... S'il n'y a pas d'argent aujourd'hui, on attendra la retraite... On descend dans la cave, on prend des pommes de terres et on les mange. J'ai 60 ans et j'ai travaillé comme trayeuse au kolkhoze toute ma vie. On travaillait à la main. Maintenant, c'est facile... J'avais 58 vaches et je travaillais dur: trois traites par jour en été et deux en 
hiver. Il n'y a plus de vache ici, elles sont dans la ferme du village d'à côté [...]. C’est quand la radiation est arrivée, notre kolkhoze a été fermé [...]. Les mauvaises vaches ont été tuées pour la viande. [Actuellement] j’ai ma vache, on en a quatre dans notre partie du village, il en a huit en tout pour 20 familles avec 4 petits enfants et des adolescents [...]. A la pharmacie il n'y a toujours rien, il n'y a pas de médicament pour se soigner, même pour la grippe! Cette année, on n’a eu rien. Avant, on nous distribuait des vêtements, des produits. En automne, on n'a eu rien. Le camion a été déchargé dans le village voisin et il n’y avait pas d'aide humanitaire pour nous [...]. C'est à cause de notre pouvoir local. Quand les démolisseurs ${ }^{11}$ viennent, ils vont et viennent et détruisent systématiquement des maisons. Les miliciens savent qu'ils emmènent les maisons en fonction de la demande. Mais il n'y a pas de contrôle pour eux et c'est interdit pour nous d'aller y prendre du bois. Alors, les gens n'y vont qu'à à travers les champs pour ne pas se faire arrêter. Chacun se débrouille. Mais si j'y vais, moi, ils m'arrêtent alors qu'un camion chargé du bois, il passe avec des maisons entières. Ils viennent avec des camions et puis repartent je ne sais où. Les démolisseurs gagnent plus d'argent en vendant les maisons qu'en les démolissant. Car c'est du bois massif et il y a toujours des acheteurs [...]. Ici, on chauffe avec du bois Mais il y a de la radioactivité dans le bois. Il y a un homme qui est venu prendre les mesures. Il a contrôlé et dans le poêle le niveau était très élevé. Le bois vient des forêts environnantes. Mais il faut bien chauffer en hiver, il n'y a pas d'autre solution [...]. Nous mangeons des champignons. ${ }^{12} \mathrm{~J}$ 'ai un baril entier de champignons salés. Parce qu'il faut manger. Si tu ne manges pas, tu crèves. Ils sont radioactifs, mais on les mange. Il faut manger, on les prépare bien, et, s'il faut, on met avec une bouteille de vodka sur la table. On dit qu'elle protège. Oui, même les médecins ont dit qu'elle protège. Il faut obligatoirement boire 100 grammes, ${ }^{13}$ ça protège [...]. Pour ma part, je me suis déjà habituée aux radiations. Mais j’ai mal aux mains, aux articulations, à la colonne vertébrale et j'ai mal à la tête. De temps en temps, j’ai l'impression qu'on me serre des tempes, surtout quand le temps change. Je pense que ça ne changera plus, que Dieu ne nous donnera plus rien de bon. On vit comme on vit. On doit vivre avec la radiation. Si j’avais déménagé dans un endroit à l'air pur, je serais morte. On s'est déjà habitué à la radiation. L’air pur est dangereux pour nous. Même les médecins disent que si nous voulons prolonger nos vies, on

\footnotetext{
${ }^{11}$ Les autorités font raser et enterrer les maisons qui sont contaminées.

${ }^{12}$ Les champignons constituent une partie importante de l'alimentation de base des habitants des campagnes du Bélarus. Le champignon est un aliment qui concentre très fortement la radioactivité.

${ }^{13}$ Cent grammes équivalent à dix centilitres.
} 
doit rester ici. C’est vrai. Depuis qu'il y a la radioactivité, il y a déjà douze ans, que je ne vais nulle part. L’année dernière, je suis parti à Sotich [ville située en Russie] i pour voir ma fille et j'ai eu très mal à la tête. J'ai eu très mal. La tension. Je suis allée voir le médecin, ils m’ont tout de suite demandé d’où je venais. J'ai dis que je venais de Biélorussie et ils m’ont tout de suite renvoyé. Ils m’ont conseillé de vite repartir chez moi, afin que je ne meure pas là-bas. Ils m'ont dis que je me suis habituée à la radiation, alors qu’à Sotchi l'air est pur.

Le départ annoncé ne vient pas, les partants sont remplacés, les mesures d'enfouissements sont contournées avec l'aval des services de milice, le bois de construction contaminé entre dans le réseau commercial contrairement à ce que ses statuts, physique et symbolique, de déchet radioactif devraient lui conférer. La contamination environnementale est bien incorporée par l'alimentation ou le chauffage au bois à base de produits prélevés dans l'environnement immédiat. Ainsi, les activités de la vie sociale ordinaire, considérées comme des facteurs d'intégration sociale sont, dans le cadre social de la vie en zone très contaminée, des agents d'amplification du développement des sociopathologies. Ainsi, la forme de rationalité qu'il y a à considérer la propriété d'un lopin comme une garantie contre la pénurie alimentaire, l'attachement au sol et à son cadre social résiste à l'attrait du départ de la zone contaminée pour une région “où l'air est pur” est un gage d'éloignement du danger. La confrontation entre l'imaginaire d'une réalité pathogène et maîtrisée d'une part et celui d'une vie sociale assujettie aux tourments et à l'instabilité loin du village d'origine d'autre part aboutit au choix de la stabilité. Ce choix ne semble pas faire pourtant illusion pour cette femme qui accepte avec résignation le diagnostic de médecin qui lui intime l'ordre de retour en zone polluée alors qu'elle en était dégagée. D’ailleurs l'addiction aux radiations résonne, dans les zones contaminées, comme une rumeur qui stigmatise les individus jusqu'à ce qu'ils retournent l'effet péjoratif pour le positiver.

\section{Conclusion}

La sociopathologie n’est pas une catégorie supplémentaire. Il n’y a pas de socio-malades mais il y a bien des personnes soumises à des processus pathogènes inscrits dans une dynamique environnementale instituée. Dans le cas de Tchernobyl, la réalité de l'exposition à la radioactivité ne souffre pas de contestation mais elle est interprétée différemment, selon l'OMS, l’AIEA ou 
les habitants. Il existe bien de nombreuses entrées dans la sociopathologie. ${ }^{14}$ En croisant, la quotidienneté des habitants avec les mesures nationales ou internationales prises dans la démarche gestionnaire des conséquences de Tchernobyl, nous avons cherché mettre en évidence les dynamiques d'incorporations pathogènes auxquelles les habitants sont exposés. Elles relèvent à la fois des principes scientifique, technocratique, économique, politique que social et sanitaire.

La sociopathologie n'est pas un concept ayant vocation à appréhender une somme de pathologies individuelles. La sociopathologie est interrogée comme un fait social. De ce point de vue cher à Durkheim (1981), elle peut être considérée comme normale au regard du développement de la société dans laquelle elle est observée; cette société doit être elle-même étudiée du point de vue de la phase correspondant à la détermination de la sociopathologie. Il est tentant de plagier le titre de la thèse de Canguilhem (2003) de manière allusive en présentant le "socionormal" et le sociopathologique pour souligner que, dans certaines situations, ce qui relève du sociopathologique est à considérer comme normal. La tension entre ces pôles que sont le normal et le pathologique, la santé et la maladie ou toutes relations à la norme sont fortes et il n'est pas possible de chercher à les fixer au niveau de l'individu comme être organisé mais bien au niveau des sociétés comme organisations.

Avec la sociopathologie, il est question d'incorporation pathogène. La question alors soulevée est celle de la responsabilité face à l'incorporation. Dans quelle mesure sommes-nous responsables de l'incorporation d'une pathologie dont nous sommes victime? Nous pouvons répondre. Notre réponse est caractérisée par le rapport que nous entretenons avec l'imaginaire symbolique, la rationalité et à la morale. Et la société peut proposer une toute autre réponse à cette incorporation: les scientifiques, politiques, économistes et moralistes proposeront de multiples interprétations qui s’ajusteront plus ou moins à la nôtre.

\footnotetext{
${ }^{14}$ Le "Forum Tchernobyl” qui regroupe divers appareils des Nations Unies dont l'AIEA et l'OMS et les gouvernements du Bélarus, de l’Ukraine et de la Russie vient de publier un rapport (2005) où il est question du nombre et du type de décès prévus à terme. 4000 personnes décèderont des conséquences sanitaires de l'explosion du réacteur. Ces résultats sont actuellement fortement discutés et contestés car ils marquent une fin de "l’histoire de Tchernobyl" contre l'avis de scientifiques qui prévoient des mutations génétiques dans plusieurs générations.
}

Horizontes Antropológicos, Porto Alegre, ano 12, n. 25, p. 191-216, jan./jun. 2006 
Le plus souvent les sociopathologies sont catalysées par des inégalités sociales qu'elles renforcent car elles prolifèrent sur un terrain où l'information n'est pas donnée, traduite dans les règlements, où les recours pour limiter l'exposition environnementale ne sont pas accessibles.

\section{Références}

AIEA. Rapport, 1958.

AIEA. Bulletin, avril 1987.

AIEA. One decade after Chernobyl. Summing up the consequences of the accident. Proceedings of an international conference, Vienna, 8-12 April 1996. p. 555.

ANDERS, Günther. L'obsolescence de l'homme: sur l'âme à l'époque de la deuxième révolution industrielle. Paris: Editions de l'Encyclopédie des nuisances: Editions Ivréa, 2002.

BECK Ulrich. The anthropological shock: Chernobyl and the contours of the risk society. Berkeley Journal of Sociology, n. 32, p. 153-165, 1987.

BECK, Ulrich. La société du risque: sur la voie d'une autre modernité. Paris: Aubier, 2001.

BELPOMME, Dominique. Ces maladies créées par l'homme: comment la dégradation de l'environnement met en péril notre santé. En collaboration avec Bernard Pascuito. Paris: Albin Michel, 2004.

BOCÉNO, Laurent. Sociopathologies: de Tchernobyl à La Hague. Thèse de Sociologie, Université de Caen Basse-Normandie, 2005.

CANGUILHEM, Georges. Le normal et le pathologique, augmenté de Nouvelles réflexions concernant le normal et le pathologique. 9ème ed. Paris, PUF/Quadrige, 2003. (Réédition de Essai sur quelques problèmes concernant le normal et le pathologique, 1943).

DOZON, Jean-Pierre. Quatre modèles de prévention. In: DOZON, J.-P. ; FASSIN, D. (Dir.). Critique de la santé publique: une approche anthropologique. Paris: Editions Balland, 2001. p. 23-46.

DURKHEIM. Emile. Les formes élémentaires de la vie religieuse. Paris: PUF, 1960. 
DURKHEIM. Emile. Les règles de la méthode sociologique. Paris: PUF, 1981.

FASSIN, Didier. Au cœur de la cité salubre: la santé publique entre les mots et les choses. In: DOZON, J.-P.; FASSIN, D. (Dir.). Critique de la santé publique: une approche anthropologique. Paris: Editions Balland, 2001. p. 47-73.

FERNEX, Michel. La catastrophe de Tchernobyl et la santé. In: GOUJON, A.; LALLEMAND, J.-C.; SYMANIEC, V. (Dir.). Chroniques sur la Biélorussie contemporaine. Paris: L'Harmattan, 2001. p. 243-278.

GERIRAD (GEstion du RIsque RADiologique). Rapport. Holland University (LDES); Université de Genève (LASAR); Université de Caen, 1998. 78 p.

HERZLICH, Claudine. [Entretien avec Stéfan Jaffrin, Dossier La maladie Fait Culturel, La santé de la molécule la sociétée. 1991. Disponible en: <http:/ /www.cybertribes.com/sante9.html>. Accès en 10 déc. 2005.

ILLICH, Ivan. Némésis médicale: l'expropriation de la santé. Paris: Seuil, 1975.

JONAS, Hans, Le principe responsabilité. Paris: Cerf, 1990.

JUAN, Salvador. La société inhumaine: mal vivre dans le bien-être. Paris:L'Harmattan, 2001. (Collection Sociologies et Environnement).

KLEINMAN, Arthur. Patients and healers in the context of culture: an exploration of the borderland between anthropology medicine and psychiatry. Berkeley: University of California Press, 1980.

KLEINMAN, Arthur. The illness narratives sufflering and healing and the human condition. New York: Basics books, 1988.

LAGADEC, Patrick. La civilisation du risque: catastrophes technologiques et responsabilité sociale. Paris: Seuil, 1981.

LEFORT, Claude. L'invention démocratique: les limites de la domination totalitaire, Paris: Editions Fayard, 1981. (Collection Biblio Essais).

LENOIR, Yves. Tchernobyl: l'optimisation d'une tragédie. Ecologie et Politique, n. 18-19, p. 11-45, 1996.

MAUSS, Marcel. Effet physique chez l'individu de l'idée de la mort suggérée par la collectivité. Journal de Psychologie Normale et Pathologie, 1926.

MAUSS, Marcel. Sociologie et anthropologie. Paris: PUF, 1968. 
OMS. Effets génétiques des radiations chez l'homme. Rapport d'un groupe d'étude réuni par l'OMS, OMS, Palais des Nations. Genève, 1957. 183 p.

OMS. Rapport d'un groupe d'étude: questions de santé mentale que pose l'utilisation de l'énergie atomique à des fins pacifiques. Série de Rapports Techniques, n. 151, OMS. Genève, 1958. p. 59.

RIEUSSET-LEMARIÉ, Isabelle. Une fin de siècle épidémique. Arles: Acte Sud, 1992. 